\title{
Characterization of Physiological Networks in Sleep Apnea Patients Using Artificial Neural Networks for Granger Causality Computation
}

\author{
Jhon Cárdenas ${ }^{\mathrm{a}}$, Alvaro D. Orjuela-Cañón ${ }^{\mathrm{a}}$, Alexander Cerquera $^{\mathrm{b}}$, and Antonio Ravelo ${ }^{\mathrm{c}}$ \\ aUniversidad Antonio Nariño. Facultad de Ingeniería Electrónica y Biomédica, Bogotá Colombia. \\ bJ. Crayton Pruitt Family Departament of Biomedical Engineeting. University of Florida,Gainesville, \\ FL, USA. \\ ${ }^{\mathrm{C}}$ Institute for Technological Development and Innovation in Communications, Universidad de Las \\ Palmas de Gran Canaria, Las Palmas de Gran Canaria 35017, Spain.
}

\begin{abstract}
Different studies have used Transfer Entropy (TE) and Granger Causality (GC) computation to quantify interconnection between physiological systems. These methods have disadvantages in parametrization and availability in analytic formulas to evaluate the significance of the results. Other inconvenience is related with the assumptions in the distribution of the models generated from the data. In this document, the authors present a way to measure the causality that connect the Central Nervous System (CNS) and the Cardiac System (CS) in people diagnosed with obstructive sleep apnea syndrome (OSA) before and during treatment with continuous positive air pressure (CPAP). For this purpose, artificial neural networks were used to obtain models for GC computation, based on time series of normalized powers calculated from electrocardiography (EKG) and electroencephalography (EEG) signals recorded in polysomnography (PSG) studies.
\end{abstract}

Keywords: Artificial Neural Networks, Granger Causality, Obstructive Sleep Apnea Syndrome, Brain-Heart Networks, CPAP.

\section{INTRODUCTION}

One of the ways that our body has to regulate different functions is through sleep, which is one of the most important neurological states since it allows the body to regenerate and restore mental and physiological aspects after a routine has been done. Sleep is basically characterized as a resting state in which the consciousness processes are diminished, while various physiological systems, controlled by the Central Nervous System (CNS), are still working in their vegetative functions, such as the respiratory system and the Cardiac System (CS). However, there are sleep pathologies in which these functions are affected, causing side effects like drowsiness, cognitive deficiencies, mood changes and lack of focus $[1][2]$.

The most common pathologies that are presented in sleep intervals are associated to respiratory disorders, such as apnea (reduction in respiratory flux larger than $90 \%$ by at least 10 seconds) and hypopnea (reduction in respiratory flux larger than $30-50 \%$ by at least 10 seconds, depending on the desaturation level in the oxygen level), being the first most recognized as obstructive sleep apnea syndrome (OSA) [3]. According to a recent report from the Spanish Society of Pneumology and Thoracic Surgery (SEPAR), OSA affects over $25 \%$ of the adult population and $6 \%$ of children, warning that in 2026 it will be the most common respiratory disease [4].

There is a set of treatments for OSA in children and adults [5][6], the most common method to treat this pathology is continuous positive air pressure CPAP, which is based on pumping air under pressure inside the airway of the lungs, holding the windpipe open through the sleep process and therefore prevent respiratory breakdowns featured in this pathology. However, it is still deeply unknown the effects on the dynamic regarding the interaction between CNS and CS as a result of the treatment, especially, regarding the changes in the information stream among systems. In the literature, there are some works that have applied tests based on Transfer entropy (TE) and linear models as Ganger Causality (GC), as most important methods. These studies have a focus in the inclusion of physiological networks for the brain-heart interconnections analysis, using electroencephalography (EEG) and electrocardiography (EKG) signals acquired during 
sleep phases, this technique is called polysomnography (PSG). In addition to recording of the described signals also registers electrooculography, airflow during respiration, oxygen saturation, and other measures are obtained [7][8].

Examples of analysis of the brain-hearth physiological networks can be found in works like the developed by Faes et al, where TE calculations were applied to quantify the transmitted information in bidirectional ways between the CNS and cardiac system in with healthy people, during sleep [9]. Likewise, under the same point of view the transmitted information was measured between CNS subsystems, assuming that each of the EEG sub-bands $(\delta, \theta, \alpha, \beta, \gamma)$ represents a subsystem of the nervous system. The authors of this work found out that the $\beta$ waves are the node that sends the majority of the information to the cardiac system that flows in the subnet brain-brain and, at the same time, transmits to the other brain subsystems the information that arrives from the cardiac system.

Jurysta et al, carried out a study applying linear measures of connectivity to predict changes in $\delta$ activity (brain sub-band) [10]. Modifications in the heart activity was characterized by the RR intervals in normal patients, and reading of visual inspection revealed that the fluctuations of high frequency of the CS (HF reflect cardiac parasympathetic activity) are related with $\delta$ frequency band of the brain signals. In 2006, this analysis was extended to male patients diagnosed with sleep apnea, reveling that the respiratory disorders in the sleep, affect the fluctuations of the heart activity [11]. This affectation is reflected by anomalies in the relation between the sympathetic cardiac modulation, the vagal modulation and the $\delta$ band subsystem. Afterwards, the suggested method by the authors was applied in the study of this type of data in patients with chronic insomnia [12]. Patients showed a reduction of coherence between the heart activity determined by the RR intervals and the $\delta$ activity on the EEG regarding normal patients. This study determined that there are changes between the autonomous heart activity and $\delta$ activity in patients with chronic insomnia, even in the absence of modifications of heart variability and other cardiac anomalies.

Most of the studies have been based on methods that present inconveniences at the moment of analyzing a group of data. For example, the linear models are established only to consider data that presents said linearity, and parametric models as the GC hold strong disadvantages [16]. One of them is the statistic supposition in the data, such as normal distribution with uniform variance. Finally, there are non-parametric methods like TE, but in this method the analytical formulas to evaluate the significance of the results are lacking and the computational cost is very high. Therefore, in this document we present a method to quantify the connection between systems combining neural networks and GC with the aim of overcoming the mentioned disadvantages.

\section{MATERIALS AND METHODS}

\subsection{Data Base}

This work was carried out with the database described by Cerquera et al [27], corresponding to polysomnographic register to 28 subjects diagnosed with OSA which were ranged in ages between 34 and 86 years and were treated with CPAP. 10 control subjects were included to determine characteristics between these groups. The registers contains only the II channel of EKG and 4 different derivations of the EEG (O2-A1, C4-A1, C3-A2, O1-A2), sampled at 200Hz and recorded in one night, where it was divided into intervals implementing the treatment and without it. Each brain band $(\delta, \theta, \alpha, \beta, \gamma)$ was considered as a subsystem of the central nervous system. The low frequency (LF, have a dominant sympathetic activity) and high frequency (HF) of the heart rate variability (HRV) was considered as subsystems of the cardiac system.

\subsection{Preprocessing}

The preprocessing employed was the same presented by Jurysta [10] and Faes [13], which is briefly explained as follows: with respect to EEG preprocessing, the channel C3-A2 was chosen to develop the EEG analysis. For each patient, the complete time series of EEG was processed to eliminate the accumulative effects in the data groups (linear detrended), and in that way demonstrate only the absolute changes in the values and allow that the potential cyclic behaviors were identified, Additionally of being filtered and a Fast Fourier Transform (FFT) was applied each 5 consecutive seconds to the window filtered EEG data, where it was calculated the strength of each sub-band EEG using trapezoidal integration. Following this, the mean of the power of each subband was calculated in non-overlapping epochs of 30 seconds and normalized with the power of the corresponding sub-band in whole recording. 
Taking into account the Pan-Tompkins algorithm [14], for each EKG time series it subsampled at $400 \mathrm{~Hz}$ to improve the detection of the R peaks, applying said algorithm. Following this, there was a subdivision in consecutive windows of 60 seconds each overlapped at half. For each window, the intervals RR were processed to eliminate the effects of the accumulation of data collection, and afterwards with the FFT to obtain its power spectrum, where the spectrum in HF and LF stabilized at the total strength of the heart rate variability $(0.04-0.15 \mathrm{~Hz}$ to $\mathrm{LF}, 0.15-0.4 \mathrm{~Hz}$ to $\mathrm{HF})$ in all recordings.

\subsection{Granger Causality and Artificial Neural Networks}

According with Montalto et al, there are different methods to quantify information between brain-heart physiological systems. One of these methods uses artificial neural networks applied in the principle of GC. This allows to achieve nonparametric analysis, and formulating a nonlinear approximation for the autoregressive interpretation [15][26].

The definition of GC is simple, it is possible to suppose two variables, $\mathrm{X}_{1}$ and $\mathrm{X}_{2}$, where the first one corresponds to the target system, and the other matches with the driver system. The latter is useful to determinate if control behavior influences to the target system. For determining these influences or causalities, a multiple time series analysis method based on autoregressive (AR) model is applied in the present work. In this case, two models are considered, where one includes information of the driver system, denominated as full model (equation 2), and a second model, which considered just information of the target system is called as reduced model (equation 1). Mathematically, it is possible to describe the systems according to:

$$
\begin{gathered}
X_{1}(t)=\sum_{j=1}^{p} A_{11, j} X_{1}(t-j)+E_{1}(t) \\
X_{1}(t)=\sum_{j=1}^{p} A_{11, j} X_{1}(t-j)+\sum_{j=1}^{p} A_{12, j} X_{2}(t-j)+E_{1}(t) \\
C=\ln \frac{\operatorname{var}\left(E^{2} R_{\text {reduced }}\right)}{\operatorname{var}\left(E_{R} R_{\text {full }}\right)}
\end{gathered}
$$

Where $X_{1}$ and $X_{2}$ are the time series describing the target and control systems, $p$ is the order of the autoregressive model, $A$ is the matrix with the coefficients of the model, and $E_{l}$ is a matrix with the forecasting residues. It is evident that the VAR model is linear because the traditional way to obtain the coefficient matrix $A$ is a linear method presented by GrangerWeiner [16]. The causality measure can be computed through the log ratio of reduced error variances and full error variances, using the equation 3 , where $E R R_{\text {reduced }}$ is the error of the model without information from $X_{1}$ to $X_{2}$, and $E R R_{\text {full }}$ is the error of the model considering all coefficients.

Artificial Neural Networks (ANN) are models extracted from computational intelligence, where units known as neurons are connected to conform specific architectures [17]. From different proposals, the Multilayer Perceptron (MLP) is known because its capabilities to obtain models for forecasting, in a similar way of AR does, but known as nonlinear autoregressive (NAR) models [24]. Based on this characteristic, an alternative for computing GC in a nonlinear mode, taking advantage of the ANN models [25][26]. For obtaining the NAR model, the time series were used for training ANN architecture, having as outcome an expression that can describe the behavior of the studied systems. In this way, using:

$$
\begin{gathered}
X_{1}(t)=f\left(\sum_{j=1}^{p} A_{11, j} X_{1}(t-j)+E_{1}(t)\right) \\
X_{1}(t)=f\left(\sum_{j=1}^{p} A_{11, j} X_{1}(t-j)+\sum_{j=1}^{p} A_{12, j} X_{2}(t-j)+E_{1}(t)\right)
\end{gathered}
$$

Where the $A$ matrices contain the coefficients that relate the $X_{1}$ and $X_{2}$ systems and $f$ is a nonlinear function. All other parameters are the same as (1) and (2). Then, the measurement called neural network Granger causality (NNGC) compared with (3), taking the error of the full and reduced models. NNGC was defined as: 


$$
\mathrm{NNGC}=\mathrm{ERR}_{\text {reduced }}-\mathrm{ERR}_{\text {full }}
$$

Where $E R R_{\text {reduced }}$ is the forecasting error obtained considered only the target variable and $E R R_{\text {full }}$ is the forecasting error evaluated taking into account of complete information. Finally, to determine whether causality over the subsystem is significance, we used the method of surrogate data implemented by the time-shift procedure proposed in [18, 19, and 20]. Basically, GC was calculated with the original time series, and then, disorganized versions of the same time series were obtained and the GC was computed again. The process was elaborated several times, and the estimated GC values were evaluated based on a null hypothesis test was used to analyze the distribution understand the behavior of GC. Each repetition of the original time series was moved in the time domain by a delay number selected randomly, adjusted to exclude the autocorrelation effects. All computations were developed by using MUTE toolbox, which holds parameters to obtain the NNGC [15][26].

\section{RESULTS}

The performed pre-processing to the data base can be observed in figure 1, which represents the powers of the normalized time series of one of the test subjects diagnosed with OSA before and during the treatment with CPAP, in addition to indicating each of the brain sub bands and the components of the (HRV).

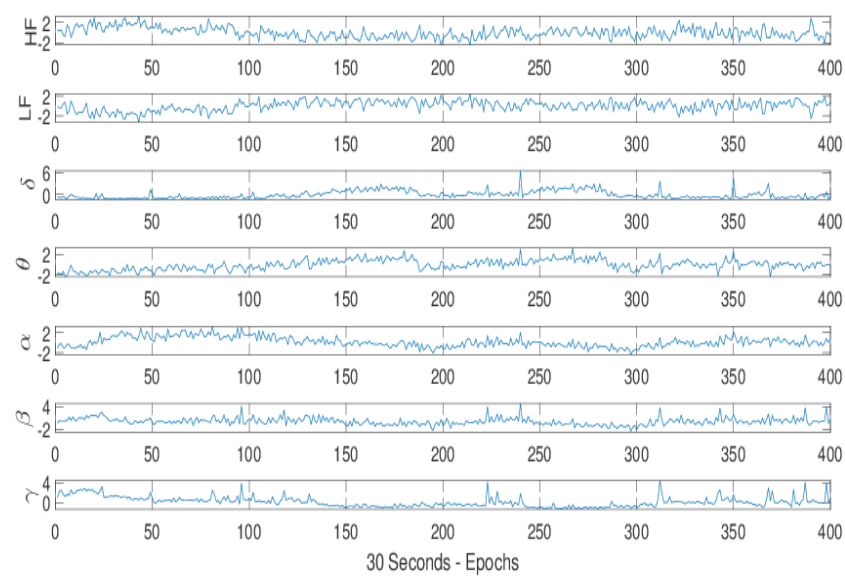

(a)

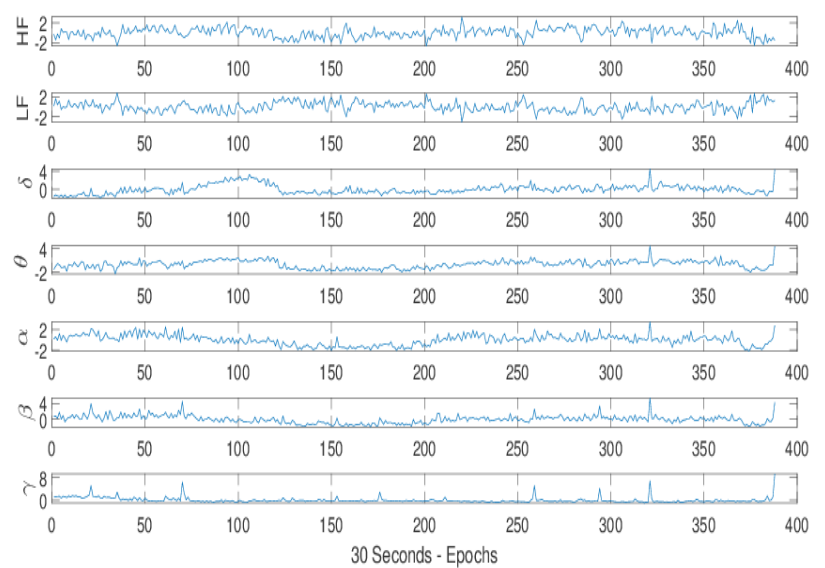

(b)

Figure 1. Time series of the normalized powers representing each of the subsystems of the brain-heart network in one of the patients of the PSG database. 1(a): before CPAP therapy; 1(b): during CPAP therapy.

For each subject under the study, according with the significance of the NNGC value, the relations between physiological systems were marked as one if there is causality and zero as opposite. Figure 2 represents the sum of these values in the set of control subjects. Gray scale was used to show amount of subjects that show causality for each systems combination. $\mathrm{Y}$-axis indicates the driver system and $\mathrm{X}$-axis the target system. For example, eighty percent of the control subjects show that HF component influences significantly in $\delta$ brain subsystem. In the same way, Figure 3 represents values for the subjects diagnosed with OSA before and during the treatment with CPAP. 


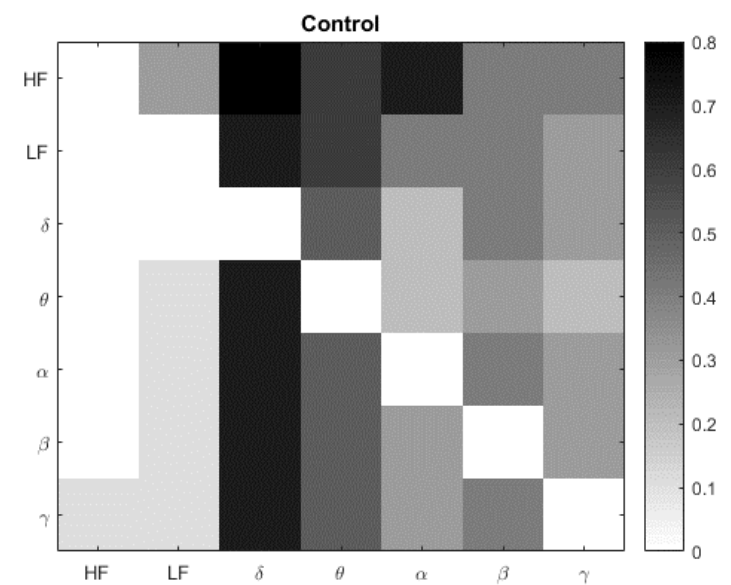

Figure 2. Amount values of the NNGC for the control subjects set.

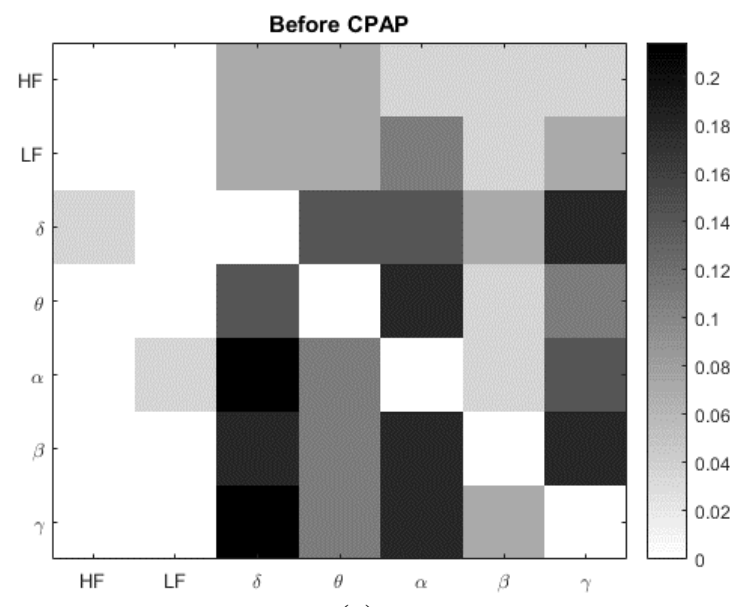

(a)

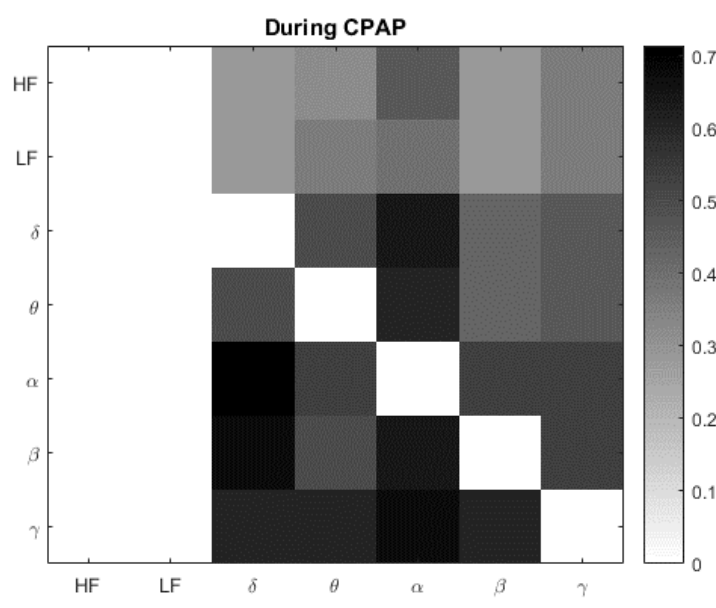

(b)

Figure 3. Amount of NNGC values for the subjects diagnosed with OSA. 3(a): before CPAP; 3(b): during CPAP.

\section{DISCUSSION}

Regarding the group of control subjects, it was possible to observe that the transmission between systems with highest values were $\beta$ band and the slow waves, mainly $\delta$ band. This is reported in previous studies as Faes et al, in [9]. The information flow from heart to brain in the $\delta$ band is in a unidirectional way. Similarly, it can be observed that brain-brain network is complexly connected, with domination of the information given by the flow of the $\delta$ band. This can be observed in the Figure $4 \mathrm{a}$, which presents the connections between systems.

Subjects with OSA presented a great decrease in the number of significant connections as can be observed in the Figure $4 \mathrm{~b}$, but it increased the information flow with other subsystems. An aspect that stands out is that the transmission of information from the $\delta$ node is no longer unidirectional between heart and brain. $\gamma$ band started to take a greater participation in the connection between systems involved, which suggests that the physiological networks between CNS and CS in apneic patients suffer a reorganization in order to find balance once again in the parasympathetic system since OSA induces sudden augments in the vagal and sympathetic heart activity [21][28]. 
During the treatment with CPAP was observed that values of NNGC were comparable in some subsystems of control subjects group. The slow waves took more participation in the connections, but the flow of information of the CS remain low. This indicates that the treatment with CPAP reestablished some aspects of the physiological networks, but not the complete structure, which was topic of study in previous works [21]. A possible factor related with the decrease of cardiac system connection, may be the increase in the heart vagal tone induced by the CPAP, documented in previous studies [22][23]. However, future studies are needed to discover the underlying pathophysiological mechanisms and the clinical relevance of this lack of recovery in the connection in the heart dynamic.

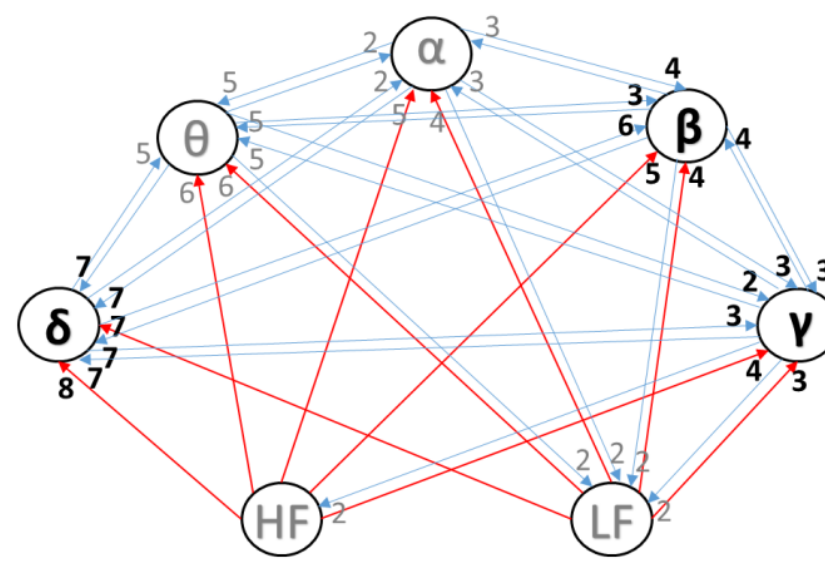

(a)

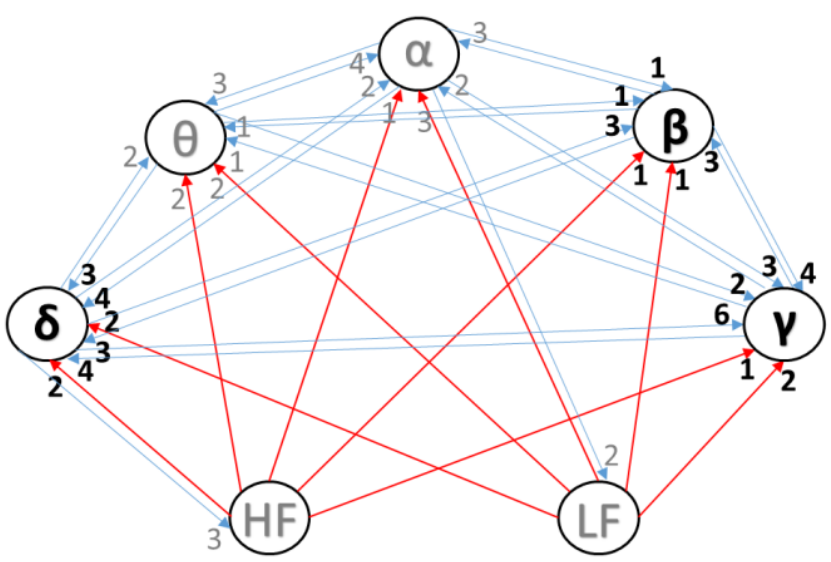

(b)

Figure 4. Connection between subsystems, the red color corresponds to the CS and the blue color to CNS. The numbers indicate the amount of subjects who demonstrated a significant connection (The number of OSA subjects were normalized to ten). 4(a): control subjects; 4(b): subject with OSA without treatment.

\section{CONCLUSION}

This study presented the used of capabilities of artificial neural networks for computing the Granger Causality. This implementation was proposed as a nonlinear analysis and determine quantitative characteristics of healthy people through physiological networks, integrated by the central nervous system and the cardiac system. Also, it was established differences with people diagnosed with OSA before and during the treatment with CPAP.

\section{REFERENCES}

[1] Yaremchuk K, Wardrop PA. [Sleep Medicine], Plural Publishing, Inc, (2010).

[2] Colten HR and Altevogt BM. "Sleep Disorders and Sleep Deprivation: An Unmet Public Health Problem", Institute of Medicine (U.S.), Committee on Sleep Medicine and Research Staff. National Academies Press, (2006).

[3] Moreno A. "Caracterización de pacientes adultos con diagnóstico de apnea del sueño". Universidad Tecnológica de Pereira - Facultad de Medicina, área de Psiquiatría. Programa de Especialización en Psiquiatría. (2012).

[4] Agüero B. Alcázar N. [Libro Blanco Neumología en España]. Respira-Fundacion Española Del Pulmon. Barcelona, (2015).

[5] R. Jane, "Engineering Sleep Disorders: From classical CPAP devices toward new intelligent adaptive ventilatory therapy" IEEE Pulse, 29 - 32, (2014).

[6] lei Wang,E. Noel,Cheung Fong, "A Wireless Sensor System for Biopotential Recording in the Treatment of Sleep Apnea Disorder”, Networking, Sensing and Control, 23-25 (2006). 
[7] Md. Zahidul Islam,K M Talha Nahiyan,Md. Adnan Kiber, “A motion detection algorithm for videopolysomnography to diagnose sleep disorder" Computer and Information Technology (ICCIT), 21 -23 (2015).

[8] M. Hirshkowitz, "Polysomnography Challenges", Sleep Medicine Clinics, 403-411, (2016).

[9] Faes L, Nollo G, Jurysta F and Marinazzo D. "Information dynamics of brain-heart physiological networks during sleep". New J. Phys 16: 1 -20, (2014)

[10] Jurysta, F., Van De Borne, P., Migeotte, P.-F., Dumont, M., Lanquart, J.-P., Degaute, J.-P., and Linkowski, P., "A study of the dynamic interactions between sleep EEG and heart rate variability in healthy young men", Clinical neurophysiology 2146-2155, (2003).

[11] Jurysta F, Lanquart JP, van de Borne P, Migeotte PF, Dumont M, Degaute JP and Linkowski P. "The link between cardiac autonomic activity and sleep delta power is altered in men with sleep apnea-hypopnea syndrome". Am J Physiol Regul Integr Comp Physiol 291: R1165-R1171, (2006).

[12] Jurysta, F., Lanquart, J. P., Sputaels, V., Dumont, M., Migeotte, P. F., Leistedt, S., ... \& Van De Borne, P. “The impact of chronic primary insomnia on the heart rate-EEG variability link". Clinical neurophysiology, 120(6), pp 1054-1060. (2009).

[13] Faes, L., Marinazzo, D., Jurysta, F., and Nollo, G., "Linear and non-linear brain-heart and brain-brain interactions during sleep," Physiological measurement 36(4), 683 (2015).

[14] Pan, J. and Tompkins, W. J., "A real-time qrs detection algorithm," IEEE transactions on biomedical engineering (3), 230-236 (1985).

[15] Montalto, A., Faes, L., and Marinazzo, D., "MuTE: a MATLAB toolbox to compare established and novel estimators of the multivariate transfer entropy," (2014).

[16] Wiener, N., "The theory of prediction", Modern Mathematics for Engineers. McGraw Hill, New York, pp. 165190 (1956).

[17] S. S. Haykin, [Neural Networks and Learning Machines], v. 10. Prentice Hall, (2009).

[18] Vlachos I, Kugiumtzis D. "Nonuniform state-space reconstruction and coupling detection". Phys Rev E 82: 016207 (2010).

[19] Quiroga RQ, Kraskov A, Kreuz T, Grassberger P. "Performance of different synchronization measures in real data: a case study on electroencephalographic signals". Phys Rev E 65: 041903. (2002).

[20] Faes L, Porta A, Nollo G. "Mutual nonlinear prediction as a tool to evaluate coupling strength and directionality in bivariate time series: comparison among different strategies based on k nearest neighbors". Phys Rev E 78: 026201, (2008).

[21] Faes L, MarinazzoD, Stramaglia S, Jurysta F,Porta A, Nollo G . "Predictability decomposition detects the impairment of brain-heart dynamical networks during sleep disorders and their recovery with treatment". (2016).

[22] Gilman MR, Floras JS, Usui K, Kaneko Y, Leung RST, Bradley TD. "Continuous positive airway pressure increases heart rate variability in heart failure patients with obstructive sleep apnea", 243-249 (2008).

[23] Nelesen RA, Yu H, Ziegler MG, Mills PJ, Clausen JL, Dimsdale JE. "Continuous positive airway pressure normalizes cardiac autonomic and hemodynamic responses to a laboratory stressor in apneic patients" 1092 1101.(2001).

[24] M. Khashei and M. Bijari, “An artificial neural network (p, d, q) model for timeseries forecasting,” Expert Syst. Appl., vol. 37, no. 1, pp. 479-489, (2010).

[25] Attanasio, A., \& Triacca, U. "Detecting human influence on climate using neural networks based Granger causality". Theoretical and Applied Climatology, 103(1-2), 103-107 (2011).

[26] Montalto, A., Stramaglia, S., Faes, L., Tessitore, G., Prevete, R., \& Marinazzo, D. "Neural networks with nonuniform embedding and explicit validation phase to assess Granger causality”. Neural Networks, 159-171. (2015).

[27] Cerquera, A., Orjuela-Cañón, A., Roa-Huertas, J., Freund, J. A., Juliá-Serdá, G., \& Ravelo-García, A. "Transfer entropy to characterize brain-heart topology in sleep apnea patients treated with continuous positive airway pressure". In 12th International Symposium on Medical Information Processing and Analysis (pp. $1016003-$ 1016003). International Society for Optics and Photonics. (2017)

[28] Jurysta, F., Kempenaers, C., Lanquart, J. P., Noseda, A., Van De Borne, P., \& Linkowski, P. "Long-term CPAP treatment partially improves the link between cardiac vagal influence and delta sleep". BMC pulmonary medicine,pp 29. (2013). 